\title{
Osteotomien und Plattenosteosynthesen am proximalen Femur bei posttraumatischen Fehlstellungen
}

\author{
R. Rahmanzadeh, M. Faensen
}

\section{Zusammenfassung}

Die Winkelplatten mit dem dazugehörenden Instrumentarium stellen die Implantate der Wahl für Korrekturosteotomien am proximalen Femur dar. Die unterschiedlichen Plattenwinkel gestatten die Korrektur auch komplexer Fehlstellungen. Nach einer exakten Planung mit Hilfe von Röntgenbildern, Pausen und Schablonen kann die erforderliche Korrekturostetomie durchgeführt und mit einer teilbelastbaren Osteosynthese stabilisiert werden.

\section{Einleitung}

Die biomechanischen Bedingungen am proximalen Femur begünstigen die Entstehung von Frakturen und stellen gleichzeitig ein erhöhtes Risiko für die Frakturheilung dar.

Pauwels (8) beschreibt die auf den Hüftkopf einwirkenden Druck- und Scherkräfte bei Schenkelhalsfrakturen in Abhängigkeit vom Frakturlinienverlauf und deren Auswirkungen auf die Dislokation und die Frakturheilung. Bei steilem, vertikalem Frakturlinienverlauf überwiegen die Scherkräfte, die der knöchernen Heilung entgegenwirken, während bei flachem, horizontal verlaufendem Frakturverlauf Druckkräfte überwiegen, die die zur Frakturheilung erforderliche Ruhe im Frakturspalt fördern.

Bei den per- und subtrochanteren Frakturen sind es ebenfalls die anatomischen Gegebenheiten mit der Lastübertragung über den Schenkelhals, die zu großen Biege-, Zug-, und Druckkräften führen. Bei

OP-JOURNAL 2000; 16: 51-55

(C) Georg Thieme Verlag Stuttgart · New York diesen extrakapsulären Frakturen wird mit großer Wahrscheinlichkeit eine Frakturheilung erreicht, doch sind Fehlstellungen, meistens im Varussinn keine Seltenheit. den Druck-, Zug- und Scherkräfte bedeuten, dass an die Osteosynthese bei proximalen Femurfrakturen besondere Ansprüche zu stellen sind, denn eine Ausschaltung dieser Kräfte durch Entlastung ist nur in sehr geringem Umfang möglich. Die zur Osteosynthese benutzten Implantate müssen die Scher- und Zugkräfte neutralisieren oder in Druckkräfte umwandeln und gleichzeitig die großen Kräfte bei Flexion und Extension auffangen. Diese Anforderung ist besonders bei instabilen Frakturen der Trochanterregion wesentlich. Bei diesen Indikationen wiesen die starren Winkelplatten Nachteile auf. Das häufige Zusammensintern der Fragmente konnte zu einer Perforation der Plattenklinge in das Gelenk füh-
Diese an der Hüfte beispielhaft auftreten-

ren, wodurch eine Reoperation mit Plattenwechsel erforderlich wurde. Bei fehlender medialer Abstützung traten nicht selten Plattenbrüche auf.

Für die Versorgung frischer Frakturen haben die Winkelplatten deshalb ihre Bedeutung verloren und wurden durch dynamische extra- oder intramedulläre Implantate verdrängt.

Die biomechanischen Erfordernissen besser angepassten Implantate haben die Zahl der Komplikationen, die nach Osteosynthesen eine Korrektur- bzw. Umlagerungsosteotomie erforderlich machten, seltener gemacht. Dennoch sind Korrekturosteotomien auch bei posttraumatischen Zuständen unentbehrlich geblieben, weil die Wiederherstellung des eigenen Hüftgelenkes, besonders bei jüngeren Patienten, anzustreben ist, auch wenn die heutigen Möglichkeiten des Hüftgelenkersatzes dazu verführen, diesen meistens leichteren Weg zu wählen.

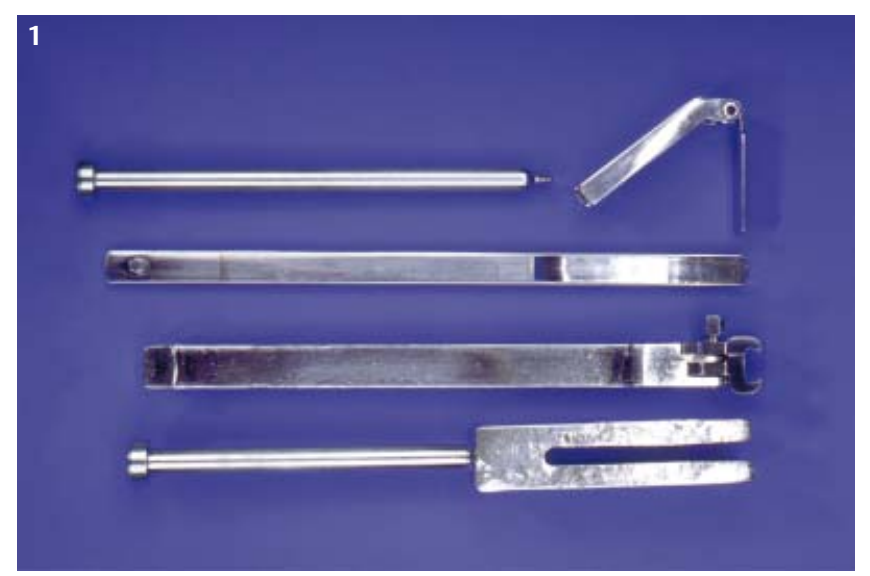

Abb. 1 u. 2 Planung und Durchführung einer valgisierenden Osteotomie bei Schenkelhalspseudarthrose.

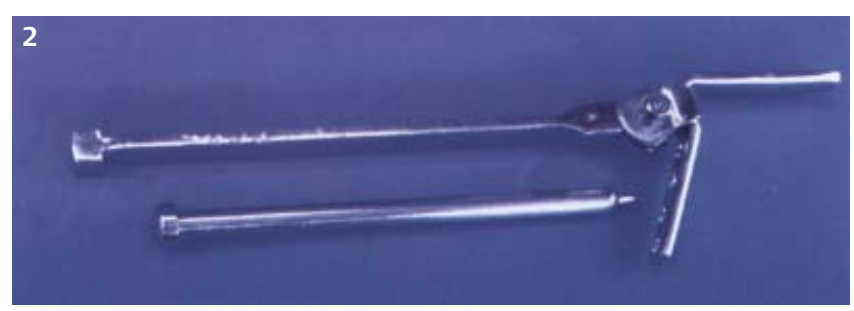




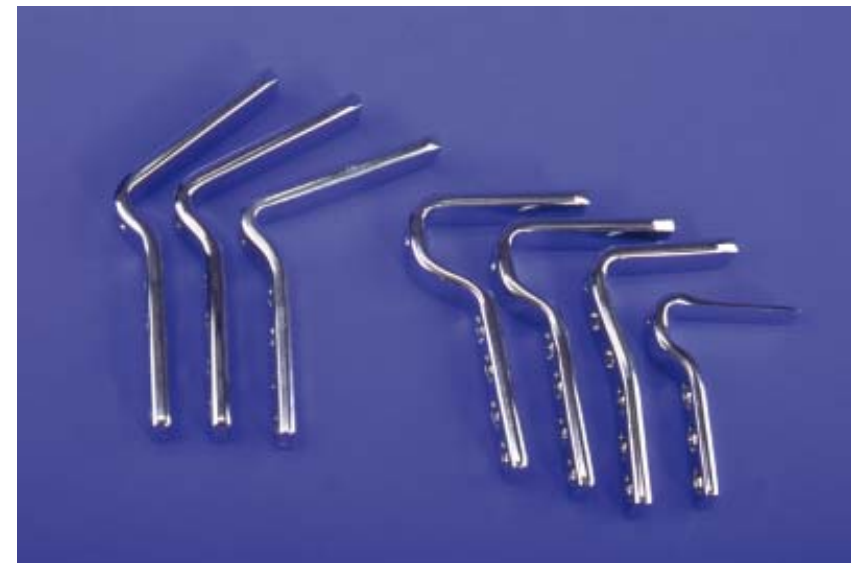

Abb. 3 Das Plattensitzinstrument kann mit dem Schlitzhammer geführt werden. Die aufgesteckte Führungsplatte erlaubt die Kontrolle beim Einschlagen des Instrumentes bezüglich der Extension und Flexion. Für die Kontrolle der Varus- und Valgusposition und der Rotation sind Bohrdrähte geeignet, die mit Hilfe der Dreieckzielplatten (Abb. 4) eingebracht werden.

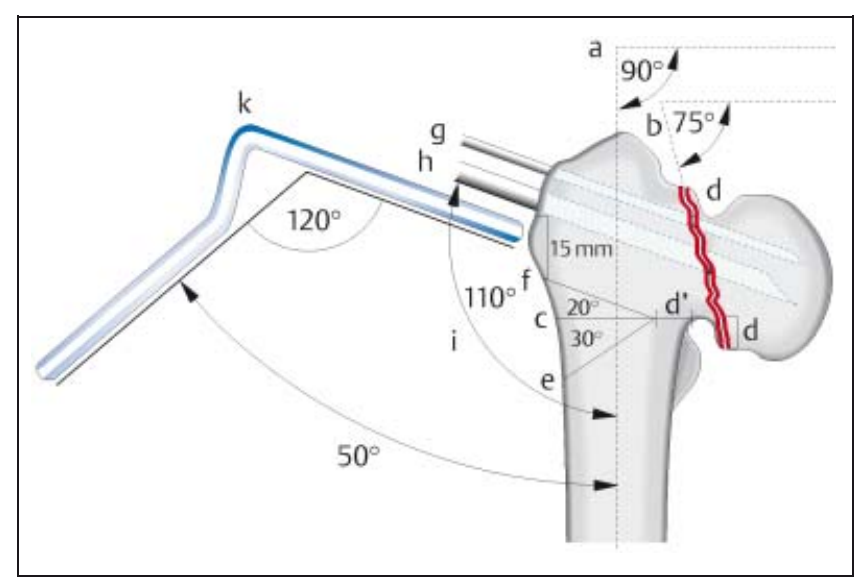

Abb. 5 Nach dem Entfernen des Plattensitzgerätes mit dem Schlitzhammer kann die im Ein- und Ausschlaginstrument fixierte Hüftplatte in das vorbereitete Plattenlager eingeschoben und mit dem Nachschlagbolzen in die endgültige Position gebracht werden.

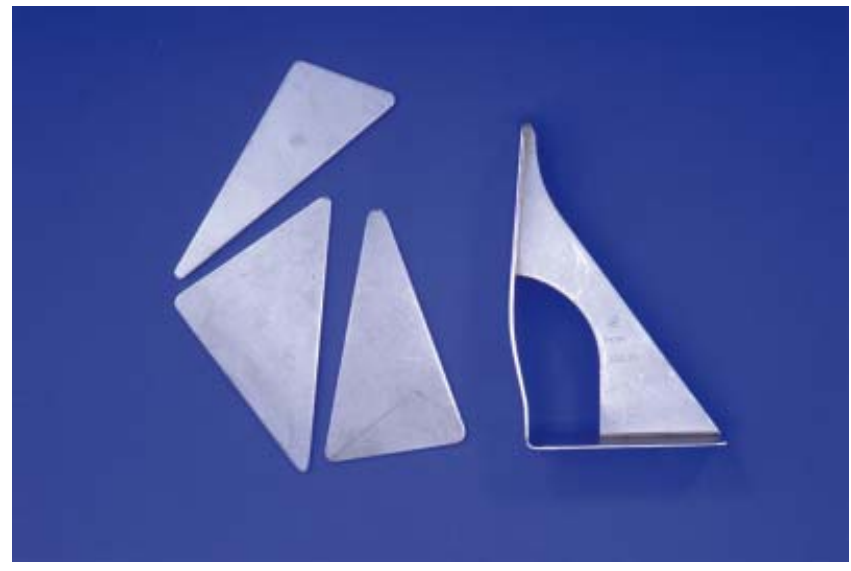

Abb. 4 Die drei Dreieckzielplatten erlauben Winkelbestimmungen von $20^{\circ}$ bis $100^{\circ}$. Das Kondylenzielgerät mit einem Winkel von $95^{\circ}$ kann am proximalen Femur durch seine Anpassung an die Anatomie korrekt angelegt werden.

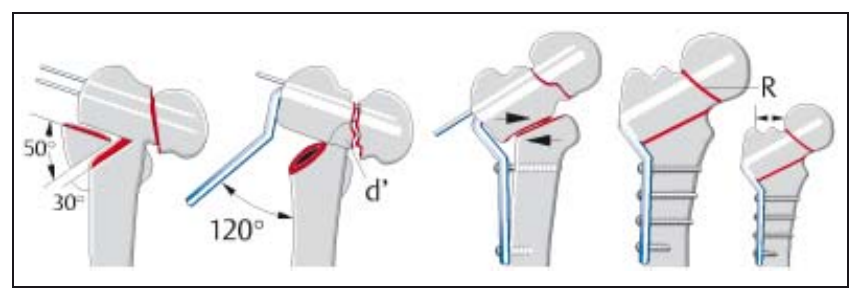

Abb. 6 Hüftplattensortiment. Für die valgisierenden Osteotomien stehen Hüftplatten mit Winkeln von $110^{\circ}, 120^{\circ}$ und $130^{\circ}$ zur Verfügung. Die Klingenlängen reichen von $50 \mathrm{~mm}$ bis $100 \mathrm{~mm}$. Die Rechtwinkelplatten mit $90^{\circ}$ und $100^{\circ}$ medialisieren durch die Tiefe ihres Bogens den Femurschaft um 10, 15 oder 20 mm. Für Jugendliche und kleine Erwachsene stehen auch kleiner dimensionierte Hüftplatten für die Varisation zur Verfügung, die an Stelle des U-Profils ein T-Profil aufweisen.
Da bei Osteotomien eine knöcherne $\mathrm{Ab}$ stützung besteht hat die Hüftplatte die Funktion einer Zuggurtungsplatte, weshalb Plattenbrüche kaum zu befürchten sind.

\section{Indikationen}

Die Inaktivitätsatrophie des Knochens, Kontrakturen der Weichteile sowie der Anspruch und die Kooperationsfähigkeit des Patienten sind bei der Indikationsstellung zu berücksichtigen.

Von den hüftnahen Frakturen sind es besonders die Schenkelhalsfrakturen, die wegen ausbleibender knöcherner Heilung bei vitalem Hüftkopf oder bei nur umschriebenen Kopfnekrosen [1] einer Umlagerungsosteotomie bedürfen. Va- rusfehlstellungen bei ungenügender Reposition oder sekundärer Dislokation stellen ebenfalls Indikationen dar. In Valgusstellung ausgeheilte Abduktionsfrakturen können besonders bei einer dysplastischen Pfanne eine präarthrotische Deformität darstellen. Bei jüngeren Patienten kann nach Ausheilung der Fraktur eine Varisierung indiziert sein, um die Belastung im Hüftgelenk zu vermindern. Gleichzeitig wird die Beinverlängerung aufgehoben. Bei frischen Frakturen mit sehr steilem Frakturverlauf (31 B2 und 3, Pauwels-3- und Garden-3und -4-Frakturen), kann eine Indikation zu einer primären Umlagerungsosteotomie bestehen $[1,6]$.

Bei Frakturen der Trochanterregion ist die Pseudarthrose selten, Fehlstellungen mit Varisierung des coxalen Femur sind häufiger. Ursachen sind die unzureichende Reposition und die von der Ideallage abweichende Position des Implantates im Schenkelhals und im Hüftkopf $[2,3]$

Bei dem sehr hohen Durchschnittsalter der Patienten mit pertrochantären Frakturen ist bei bestehender Osteopenie und fehlender Kooperationsfähigkeit der Patienten auch an einen sekundären Gelenkersatz zu denken. Die heute zur Verfügung stehenden besseren Implantate und der gelegentlich indizierte primäre Gelenkersatz lassen dieses Problem jedoch seltener werden.

Die augenfällige Varus- oder Valgusstellung kann mit anderen Fehlstellungen 

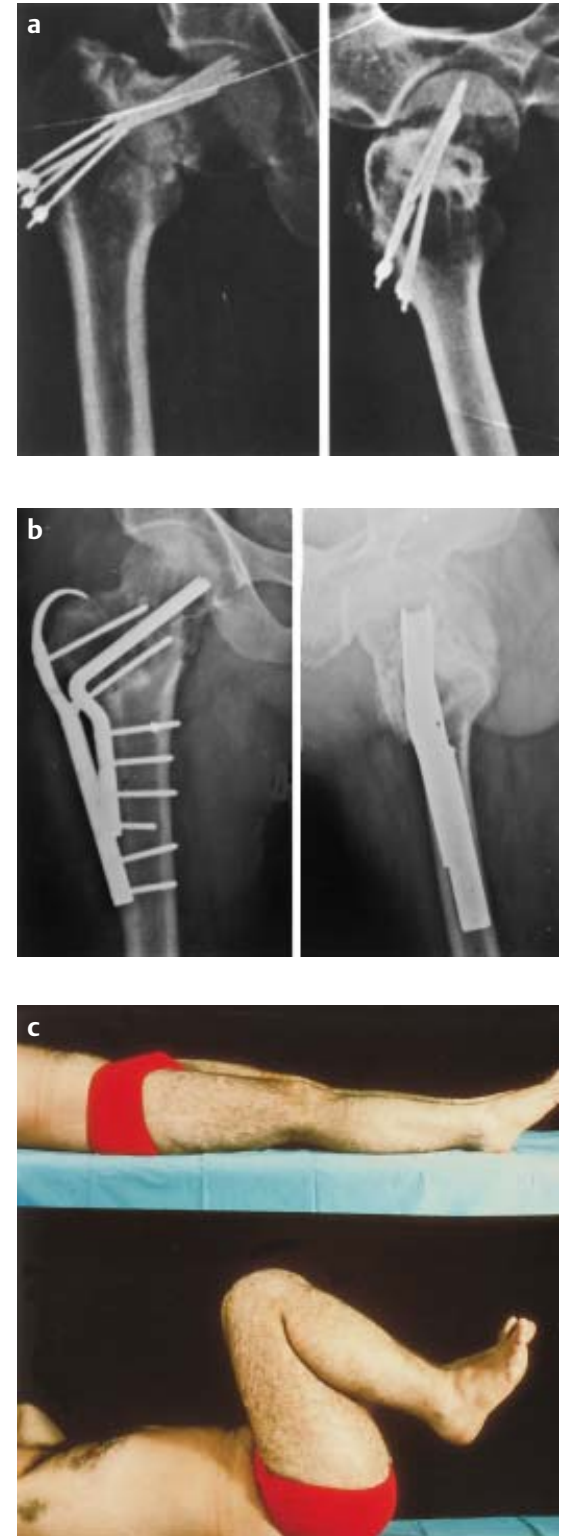

kombiniert sein, wie Extension und Flexion, Innen- und Außenrotation sowie Beinverkürzungen und selten Beinverlängerungen. Bei nicht ausgeheilten Frakturen dient die Korrekturosteotomie der Frakturheilung, bei in Fehlstellung ausgeheilten Frakturen der Wiederherstellung der physiologischen Biomechanik.

Am Hüftgelenk selbst kann die Fehlstellung zu einer Muskelinsuffizienz führen (Duchenne-Hinken, Trendelenburg, Zeichen) und zu Bewegungseinschränkungen führen. Die Auswirkung hüftnaher Fehlstellungen auf die Statik des Beines führt zu einer medialen oder lateralen Gonarthrose bei Varus- bzw. Valgusfehlstellungen mit nachfolgenden

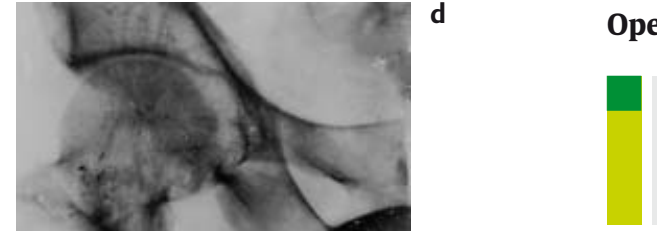

\section{Operationsplanung}

Keine Operation ohne exakte gezeichnete Planung mit Schablonen im a-p Strahlengang. Berücksichtigung von Extension / Flexion und Rotation.

Wie bei jeder Osteosynthese ist die sorgfältige Operationsplanung eine Voraussetzung für den Operationserfolg.

Bei der Korrekturosteotomie ist eine genaue Analyse der bestehenden Fehlstellung und der angestrebten Korrektur in allen Ebenen zwingend. In den meisten Fällen ist es möglich, die Anatomie der Gegenseite als Planungsziel zu benutzen.

Müller [4,5,6] hat das Winkelplatteninstrumentarium und dessen Anwendung für verschiedene Eingriffe an der Hüfte beschrieben.

Einen besonderen Vorteil stellt das Plattensitzinstrument in Verbindung mit der aufsteckbaren Führungsplatte und den Messdreiecken dar, die grundsätzlich die Durchführung der Korrektur ohne intraoperative Röntgenkontrolle ermöglichen.

Abb.7a-d Der 27-jährige Patient H. H. stellte sich mit einer Pseudarthrose bei mit Knowles-Pins versorgter medialer Schenkelhalsfraktur vor (a). Die gebrochenen Pins hatten einen ausgedehnten knöchernen Defekt erzeugt, der bei der valgisierenden Osteotomie mit der allogenen Spongiosa aus zwei Hüftköpfen aufgefüllt wurde. Die lange bestehende Beinverkürzung von $4 \mathrm{~cm}$ machte bei der Valgisierung die zusätzliche Sicherung des Trochanter major mit einer Hakenplatte erforderlich (b). Nach der Implantatentfernung 18 Monate später (d) besteht ein gutes funktionelles Ergebnis (c).

Bandinstabilitäten und Störungen im Femoropatellargelenk. Beinlängendifferenzen können zu skoliotischer Fehlhaltung und statischen Skoliosen mit folgender Osteochondrose und Spondylarthrose führen. Die biomechanischen Auswirkungen der Korrekturosteotomie gehen also über das Hüftgelenk hinaus.

Wie die Dokumentation vieler Fälle von Umstellungsosteotomien bei Coxarthrosen zeigt, ist es durchaus möglich, dass unter günstigeren biomechanischen Bedingungen eine Regeneration des Knorpels eintritt, so dass auch posttraumatische Arthrosen aufgrund einer Fehlstellung nicht grundsätzlich eine Kontraindikation für einen Korrektureingriff darstellen.
Die Implantate erlauben eine Medialisierung des Schaftes von 10, 15 und $20 \mathrm{~mm}$. Eine Lateralisierung ist mit der $160^{\circ}$-Platte möglich.

Die doppelt abgewinkelten Winkelplatten von $90^{\circ}, 100^{\circ}, 110^{\circ}, 120^{\circ}$ und $130^{\circ}$ gestatten es, die Klinge parallel mit einem Abstand von $15 \mathrm{~mm}$ zum vorgesehenen Osteotomiespalt einzubringen. Bei den posttraumatischen Korrekturen handelt es sich fast ausschließlich um Valgisierungen, gelegentlich kombiniert mit einer Extension oder Flexion. Beträgt der geplante Korrekturwinkel $30^{\circ}$ bis $60^{\circ} \mathrm{kommt}$ die $120^{\circ}$ - oder $130^{\circ}$-Winkelplatte, bei kleineren Winkeln meistens die $110^{\circ}$-Winkelplatte zur Anwendung. Die Wahl des Plattenwinkels und der Klingenlänge erfolgt mit Hilfe von Schablonen und den Röntgenbildern. Eine Pause der Röntgenbilder erlaubt die eindimensionale Simulation der Operation, indem die Keilentnahme an der Pause durchgeführt wird und das Ergebnis der Operationsplanung und die Lage der Platte kontrolliert werden können.

Die Technik der Planung wurde von M. E. Müller $[4,5,6]$ entwickelt und beschrieben. Als Beispiel wird in der Abb. 7 die Valgisation bei einer Schenkelhalspseudarthrose dargestellt. 

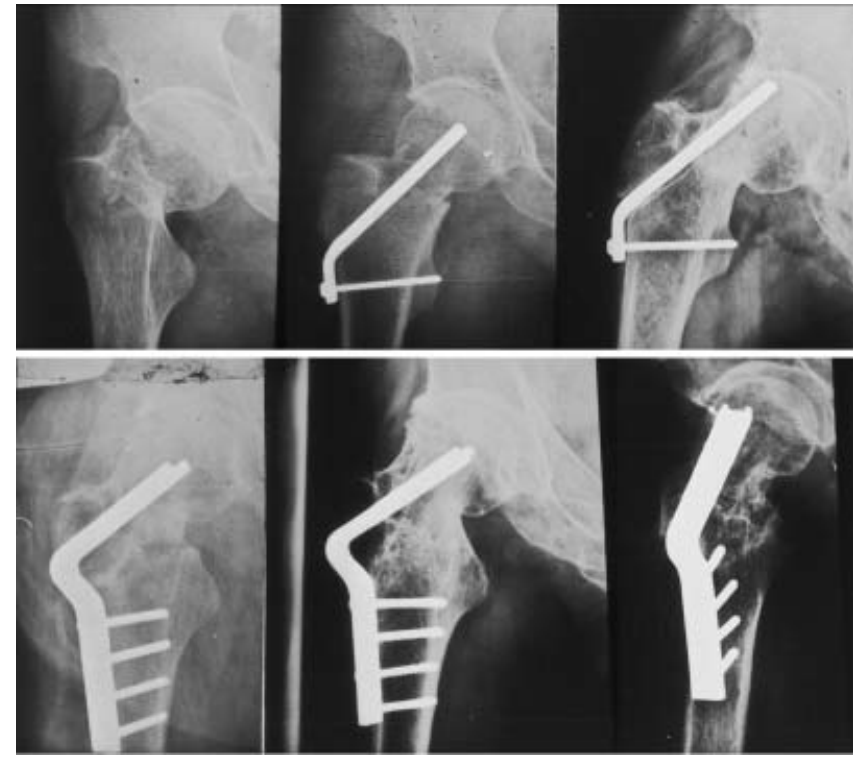

Abb. 8 Bei einem 27-jährigen Patienten G. F. bestand eine Pseudarthrose und Varusfehlstellung nach unzureichender Reposition und Osteosynthese einer medialen Schenkelhalsfraktur mit einer $130^{\circ}$-Winkelplatte. Durch die valgisierende Osteotomie mit einer $120^{\circ}$-Winkelplatte kam es zur knöchernen Ausheilung.
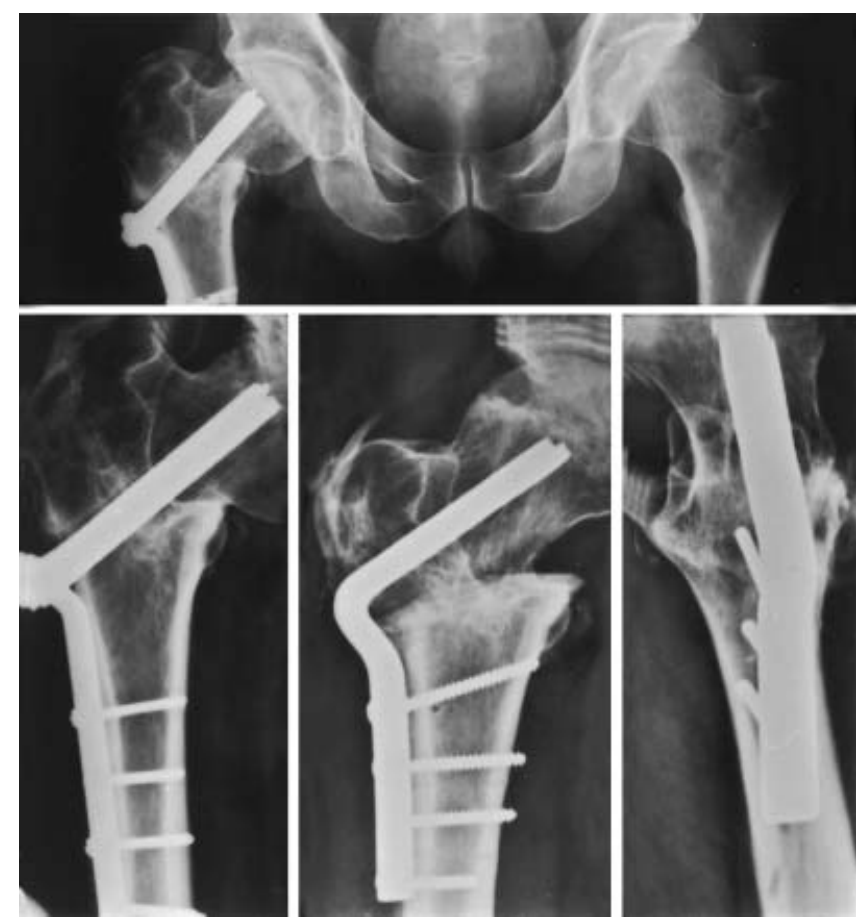

Abb. 9 Bei einem 32-jährigen Patienten B. A. kam es nach Versorgung einer pertrochanteren Femurfraktur mit einem Dreilamellen-Laschennagel zu einer Varusfehlstellung mit einem CCD-Winkel von unter $90^{\circ}$. Die knöcherne Ausheilung mit Ausgleich der Beinlänge wurde durch eine valgisierende Osteotomie mit einer $120^{\circ}$-Winkelplatte erreicht.
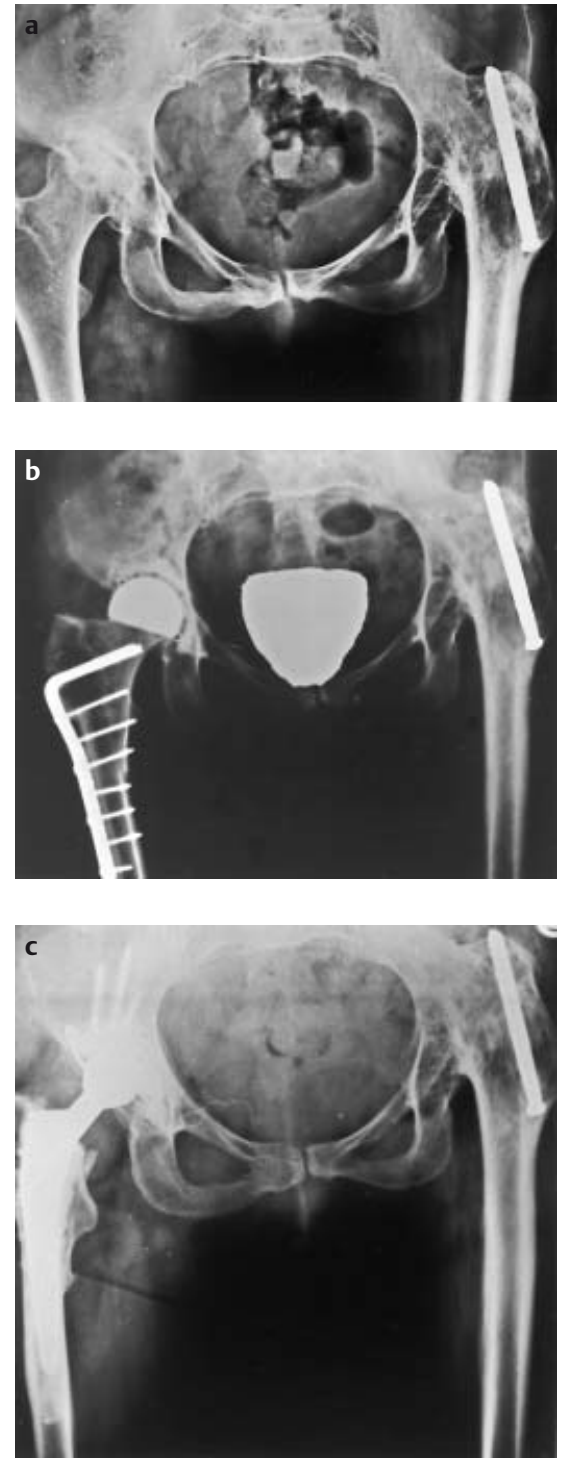

Abb.10 Eine 45-jährige Patientin M. E. mit Arthrodese des linken Hüftgelenkes stellte sich mit einer Coxarthrose zur Operation vor. Um den Beinlängenunterschied von $7 \mathrm{~cm}$ bei dem Gelenkersatz weitgehend auszugleichen, wurde eine Schalenprothese implantiert, die die gleichzeitige intertrochantere Verkürzungsosteomie erlaubte. Nach 5 Jahren entstanden Schmerzen durch die Lockerung der Prothese auf Grund einer Teilnekrose des Hüftkopfes. Jetzt konnten, dem damaligen Kenntnisstand in der Endoprothetik entsprechend, eine Geradschaftprothese und eine Pfanne mit Pfahlschrauben implantiert werden. 

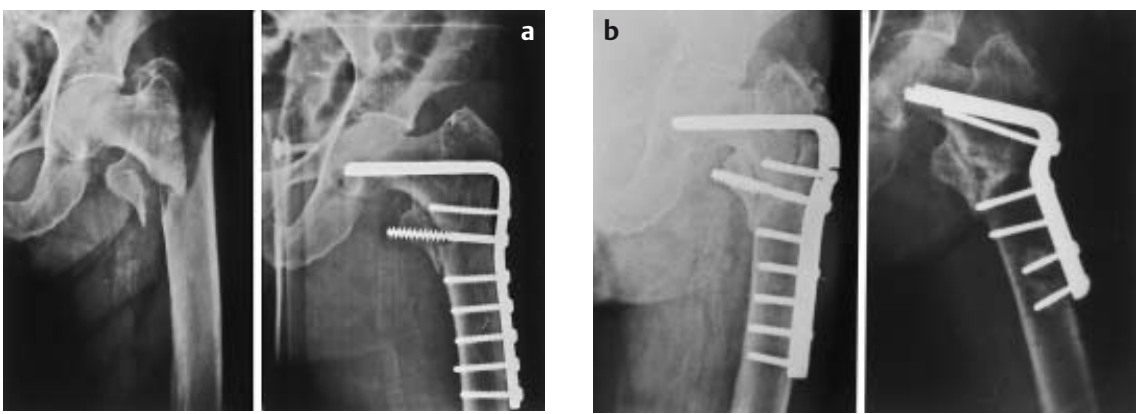

Abb.11 Eine 73-jährige Patientin H. A. mit in Varusfehlstellung mit Kondylenplatte stabilisierter pertrochanterer Fraktur (a) wurde mit einem Plattenbruch vorgestellt. Nach valgisierender Osteotomie erfolgte die knöcherne Heilung (b).

\section{Operationstechnik}

Die Röntgenbilder und Planskizzen müssen während der Operation gut sichtbar sein. In Rückenlagerung erfolgt die Abdeckung mit beweglichem Bein. Der Zugang zur Hüfte nach Watson-Jones erfolgt zusätzlich mit L-förmigem Ablösen des Musculus vastus lateralis. Die Darstellung des Schenkelhalses erleichtert die Orientierung und verhindert insbesondere, dass die Osteotomie in den Schenkelhals oder den Trochanter minor mündet.

Der entscheidende Operationsschritt ist die Platzierung des Plattensitzinstrumentes. Valgus/Varus und Flexion/Extension werden damit bestimmt. Die Rotation kann durch Drehung des distalen Fragmentes gegen das proximale beeinflusst werden. Durch Spickdrähte, die mit Hilfe der Messdreiecke eingebracht werden, können alle Operationsschritte kontrolliert werden. Die interfragmentäre Kompression wird bei Rechtwinkelplatten durch Spannen der Platte mit dem Plattenspanner und/oder den Spanngleitlöchern erzielt. Bei schrägem Verlauf des Osteotomiespaltes ist dieser so gerichtet, dass durch das Anziehen der Schrauben im Osteotomiespalt eine Kompression entsteht.

Bei der intertrochanteren Verkürzungsosteotomie kommt die von ME Müller 1971 [4] angegebene komplizierte Osteotomie mit Einstauchung des distalen Fragmentes in das proximale nicht zur Anwendung. Die intertrochantere stufenförmige Osteotomie wie sie von Schweikert [12] und Rahmanzadeh [10] angegeben wurde, weist mehrere Vorteile auf. Dieses Verfahren ist technisch einfacher, mit gleicher Technik kann auch eine Verlängerung von bis $\mathrm{zu} 4 \mathrm{~cm}$ erreicht werden, kleinere Valgus- oder Varusfehlstellungen können korrigiert werden, und sie ermöglicht eine übungsstabile Osteosynthese mit Teilbelastbarkeit.

Die Entnahme eines Zylinders aus dem proximalen Femur unter Schonung des Trochanter minor und die Osteosynthese mit einer Rechtwinkelplatte erzielt ebenfalls Übungsstabilität mit Teilbelastung [6]. Dieses Verfahren ist aber für die Verlängerung weniger geeignet.

\section{Nachbehandlung}

Die intensive Nachbehandlung kann abhängig von der Vorgeschichte mehrere Monate in Anspruch nehmen und setzt eine entsprechende Aufklärung und Bereitschaft des Patienten voraus.

Die Winkelplattenosteosynthese erlaubt eine Teilbelastung mit $20 \mathrm{~kg}$. Die Osteotomie gewährleistet im Gegensatz zu vielen Frakturen eine mediale Abstützung, so dass ein Plattenbruch nicht zu erwarten ist. Das Anheben des Beines aus der Horizontalen führt zu starken Biegekräften und soll erst nach der zu erwartenden knöchernen Heilung nach acht Wochen durchgeführt werden. Muskelkräftigung, Gehschule und Bewegungsübungen im Bewegungsbad erfolgen in Intensität und Dauer der individuellen Situation angepasst.

\section{Schlussfolgerungen}

Weiterentwickelte Marknägel und Fixateur-externe-Systeme können bei bestimmten, besonders bei multifokalen Fehlstellungen des Femurs zur Anwendung kommen. Für die Mehrzahl der Fälle stellt jedoch die Winkelplattenosteosynthese in Verbindung mit einer exakt geplanten Osteotomie ein sicheres und bewährtes Verfahren dar, das in der Hand eines geübten, nicht unbedingt spezialisierten Operateurs, mit großer Sicherheit zum Erfolg führt.

\section{Literatur}

${ }^{1}$ Ballmer FT, Ballmer PM, Mast JW, Ganz R. Ergebnisse nach Umlagerungsosteotomien bei verzögerter Heilung oder Pseudarthrose am proximalen Femur. Unfallchir 1992; 95: $511-517$

2 Baumgaertner MR, Solberg BD. Awareness of Tip - Apex Distance Reduces Failure of Fixation of Trochanteric Fractures of the Hip. J Bone Joint Surg 1997; 79-B: 969-971

${ }^{3}$ Larsson S, Friberg S, Hansson L.-I. Trochanteric Fractures, Influence of Reduction and Implant Position on Impaction and Complications. Clin Orthop 1990; 259: 130-139

${ }^{4}$ Müller ME. Die hüftnahen Femurosteotomien. Georg Thieme Verlag 1971

${ }^{5}$ Müller ME. Planung einer komplexen intertrochanteren Osteotomie. Z Orthop 1979; 117: $145-150$

${ }^{6}$ Müller ME, Allgöwer M, Schneider R, Willenegger H. Manual of Internal Fixation. Springer-Verlag 1979

7 Müller ME. Intertrochanteric Osteotomy: Indication, Preoperative Planning, Technique In: Schatzker J (ed) The Intertrochanteric Osteotomy. Springer-Verlag 1984

${ }^{8}$ Pauwels F. Der Schenkelhalsbruch, ein mechanisches Problem. in: F Pauwels: Gesammelte Abhandlungen zur funktionellen Anatomie des Bewegungapparates. SpringerVerlag 1965

9 Pauwels F. Biomechanical Principles of Varus/Valgus Intertrochanteric Osteotomy (Pauwels I and II) in the Treatment of Osteoarthritis of the Hip. in: Schatzker, J. (ed) The Intertrochanteric Osteotomy. Springer-Verlag 1984

${ }^{10}$ Rahmanzadeh $R$, Wessingage $D$, Schweikert C.-H. Die intertrochantere Verkürzungsosteotomie. actuelle traumatol 1971; 1 : $101-107$

${ }^{11}$ Rahmanzadeh R, Faensen M. Zur Korrektorosteotomie am koxalen Femurende. Akt Taumatol 1982; 12: $311-316$

12 Schneider R. Intertrochanteric Osteotomy in Osteoarthritis of the Hip Joint. in: Schatzker J. (ed) The Intertrochanteric Osteotomy. Springer-Verlag 1984

${ }^{13}$ Schweikert C-H, Rahmanzadeh R. Femurschaft- und intertrochantere Osteotomie als Behandlungsprinzip des posttraumatischen Längenunterschiedes. Hefte zur Unfallheilk 107, Springer-Verlag 1971

Univ. Prof. Dr. med. R. Rahmanzadeh Direktor der Abteilung für Unfall- und Wiederherstellungschirurgie

Universitätsklinikum Benjamin Franklin der Freien Universität Berlin Hindenburgdamm 30 12200 Berlin

Prof. Dr. med. Michael Faensen Chefarzt der Abteilung für Allgemein-, Unfall- u. Wiederherstellungschirurgie

Wenckebach-Krankenhaus

Wenckebachstr. 23

12099 Berlin

Tel.: 030/73612262 